\title{
Effect of Different Elbow Push-up plus Exercises on Upper Trapezius and Serratus Anterior Muscle Activity
}

\author{
YANG-GON $\mathrm{KIM}^{1)}$, JAE-SEOP $\mathrm{OH}^{2}$, WON-GYU $\left.\mathrm{YOO}^{2}\right)^{*}$ \\ 1) Department of Physical Therapy, Graduate School, Inje University \\ 2) Department of Physical Therapy, College of Biomedical Science and Engineering, Inje University \\ and Elderly Life Redesign Institute: 607 Obangdong, Gimhae, Gyeongsangnam-do 621-749, \\ Republic of Korea. TEL+82 55-320-3994, FAX:+82 55-329-1678
}

\begin{abstract}
Purpose] This study compared the activations of the upper trapezius (UT) and serratus anterior upper (USA) and lower fibers (LSA) during elbow push up plus exercises (EPP) with various foot placements. [Subjects] We recruited 15 male subjects. [Methods] Elbow push up plus exercises were performed on three different foot placements: the elbow push-up plus with an elbow bench (EPP-EB), the elbow push-up plus on the floor (EPP-F), and the elbow push-up plus with a foot bench (EPP-FB). Electromyography activities of the USA, LSA and UT muscles were recorded. [Results] The activations of USA and LSA muscles were significantly higher when foot placement was relatively elevated. The activation of UT during EPP-FB exercise was significantly higher than during EPP-EB exercise. [Conclusion] Foot placement in the elbow push-up plus exercise for selective activation of SA muscles must be considered by clinicians.

Key words: Elbow push up plus, Electromyography, Serratus anterior
\end{abstract}

(This article was submitted Oct. 23, 2012, and was accepted Nov. 26, 2012)

\section{INTRODUCTION}

Recently, studies of shoulder injuries have begun to focus increasing attention on the scapula ${ }^{1-3)}$. One muscle, the serratus anterior (SA), is considered to be an important stabilizer of the scapula ${ }^{4,5)}$. For this reason, researchers have devised many different exercises that promote the selective activation of the SA muscle ${ }^{6,7)}$. However, a study by Sandhu et al. ${ }^{4}$ demonstrated that an elbow push up exercise (EPP), in which the upper body height is set equal to that of a standard push up exercise, elicited greater activation of the SA and lesser activation of the upper trapezius (UT) than is produced in a standard push up exercise. In addition, performing the EPP with a Cuff Link, which is a device that allows performance of EPP exercises under unstable conditions, resulted in a significantly higher level of SA and a lower level of UT activity than in the push-up plus exercises with hand support ${ }^{8}$. Foot position is important in shoulder muscle activation during EPP exercises. During the pushup plus exercise, the foot position influences muscle activities of the adjacent shoulder $\left.{ }^{9}, 10\right)$. Lehman et al. ${ }^{9)}$ showed that SA activity increases when more body weight is located on the upper limbs with elevation of the feet during the push-up plus exercise. Therefore, foot position may also affect the activity of the SA muscle in EPP exercise. In the present study, we investigated the effects of foot position on activities of the upper SA (USA), lower SA (LSA), and UT

*To whom correspondence should be addressed. E-mail: won7y@inje.ac.kr muscles, focusing on foot elevation during an EPP exercise.

\section{SUBJECTS AND METHODS}

Fifteen male subjects (height $176.5 \pm 4.2 \mathrm{~cm}$, mass 71.4 $\pm 8.8 \mathrm{~kg}$, age $22.4 \pm 1.1$ years) took part in this study. Participants without any history of upper limb discomfort or pain within 6 months were included in this study. The EMG data were collected (sampling rate $2000 \mathrm{~Hz}$ ) using a Trigno wireless system (Delsys, Boston, MA, USA) with surface electrodes. Three electrodes were securely taped to the following muscles on the right side: USA, placed on the midaxillary line of the right side across the 5th rib; the LSA, at the 7th rib across the right lateral thorax; and UT, at approximately half of the distance between the 7 th cervical spinous and acromion processes. The EMG amplitude was normalized by collecting reference voluntary isometric contractions. The standard elbow push up plus on the floor begins with the elbows flexed to $90^{\circ}$, upper extremity weight borne on the elbows, and lower extremity weight borne on the feet in the standard manner. The participants then performed the elbow push up plus exercises with the addition of full scapular abduction (the "plus") for 5 seconds. Each participant was instructed to position the glenohumeral joint directly above the elbow, with the trunk and lower limbs held straight. Three different conditions were tested: 1) the elbow push-up plus exercise with the upper trunk elevated using a $25 \mathrm{~cm}$-high elbow bench (EPP-EB); 2) the standard elbow push-up plus exercise on the floor (EPP-F); and 3) the elbow push up plus exercise with the feet elevated using a 
$25 \mathrm{~cm}$-high foot bench (EPP-FB). SPSS v.12.0 (IBM Corp., Armonk, NY, USA) was used for statistical analysis. Oneway repeated-measures ANOVA was performed to test for differences in each of the \%RVICs of the muscle activities among in the exercises. Significant differences with pairwise multiple comparison were tested by conducting the least squares difference test to confirm specific differences among the exercises. For all three exercises, $\mathrm{p}<0.05$ was used for statistical significance.

\section{RESULTS}

The muscle activities of the upper serratus anterior was significantly increased in the order of EPP-EB (83.33 $\pm 28.37 \%)<$ EPP-F $(101.19 \pm 40.60 \%)<$ EPP-FB $(104.40$ $\pm 46.86 \%)(\mathrm{p}<0.05)$. The muscle activity of lower serratus anterior was significantly increased in EPP-F (131.44 \pm $59.37 \%)$ and EPP-FB (132.76 $\pm 55.01 \%)$ exercise compared to EPP-EB $(92.58 \pm 32.70 \%)(\mathrm{p}<0.05)$. The upper trapezius muscle was significantly increased in EPP-FB (25.23 \pm $15.16 \%)$ exercise compared to EPP-EB $(17.69 \pm 10.98 \%)(p$ $<0.05)$. The muscle activity of the upper trapezius muscle in EPP-F exercise was $20.85 \pm 13.46 \%$.

\section{DISCUSSION}

Most modified push up plus exercises are difficult to perform because the push up force passes the elbow and wrist joint during the exercise ${ }^{6}$. Therefore, elbow push-up plus exercise, excluding compensation motions of the wrist and elbow joint, was chosen for the present study. The present study showed that muscle activations of the USA and LSA were the highest in the EPP-FB exercise. The USA muscles generate scapula mobility and the LSA muscle is activated under a condition of scapular stability ${ }^{10)}$; therefore, muscle activation of both parts of the SA was increased by elevating the foot position. The reason for the observed high activities of the USA and LSA muscles in the EPP-FB exercise is that more body weight is placed on the upper body by elevating the feet during the push-up plus exercise ${ }^{9}$. Ludewig et al. ${ }^{6}$ recommended that a scapular stability exercise should have minimal effect on the UT and should stimulate the SA. The standard push up plus exercise is now the preferred exercise for activation of the SA muscle while minimizing the activity of the upper trapezius (UT) muscle ${ }^{6}$ ). The elbow push-up exercise may provide more activation of the SA, while still minimizing UT activation, compared to the standard pushup plus exercise ${ }^{8)}$. Our findings indicate that muscle activation of the UT was significantly higher during the EPP-FB exercise than during the EPP-EB exercise. We suggest that proper selection of foot height in an elbow push up plus exercise would help the activation of the SA muscles. However, excessive foot height for activation of the SA muscles may induce hypertension in the UT. Therefore, foot placement in EPP exercises aimed at selective activation of the SA muscles must be decided by the clinicians involved.

\section{ACKNOWLEDGEMENT}

This research was supported by Basic Science Research Program through the National Research Foundation of Korea (NRF) funded by the Ministry of Education, Science and Technology (No. 2012001058).

\section{REFERENCES}

1) Forthomme B, Crielaard JM, Croisier JL: Scapular positioning in athlete's shoulder: particularities, clinical measurements and implications. Sports Med, 2008, 38: 369-386. [Medline] [CrossRef]

2) Kibler WB: The role of the scapula in athletic shoulder function. Am J Sports Med, 1998, 26: 325-337. [Medline]

3) Ludewig PM, Reynolds JF: The association of scapular kinematics and glenohumeral joint pathologies. J Orthop Sports Phys Ther, 2009, 39: 90 104. [Medline]

4) Sandhu JS, Mahajan S, Shenoy S: An electromyographic analysis of shoulder muscle activation during push-up variations on stable and labile surfaces. Int J Shoulder Surg, 2008, 2: 30-35. [Medline] [CrossRef]

5) Smith R Jr, Nyquist-Battie C, Clark M, et al.: Anatomical characteristics of the upper serratus anterior: cadaver dissection. J Orthop Sports Phys Ther, 2003, 33: 449-454. [Medline]

6) Ludewig PM, Hoff MS, Osowski EE, et al.: Relative balance of serratus anterior and upper trapezius muscle activity during push-up exercises. Am J Sports Med, 2004, 32: 484-493. [Medline] [CrossRef]

7) Ekstrom RA, Bifulco KM, Lopau CJ, et al.: Comparing the function of the upper and lower parts of the serratus anterior muscle using surface electromyography. J Orthop Sports Phys Ther, 2004, 34: 235-243. [Medline]

8) Tucker WS, Armstrong CW, Gribble PA, et al.: Scapular muscle activity in overhead athletes with symptoms of secondary shoulder impingement during closed chain exercises. Arch Phys Med Rehabil, 2010, 91: 550-556. [Medline] [CrossRef]

9) Lehman GJ, MacMillan B, MacIntyre I, et al.: Shoulder muscle EMG activity during push up variations on and off a Swiss ball. Dyn Med, 2006, 5: 7. [Medline] [CrossRef]

10) Inman VT, Saunders JB, Abbot LC: Observations on the function of the shoulder joint. J Bone Joint Surg, 1944, 26A: 1-30. 